\title{
Incidence of DVT in Hip \& knee replacement patients treated by only aspirin \& mechanical device - A prospective study of 247 patients
}

\author{
Avinash C.K ${ }^{1, *}$, S. M Hashim ${ }^{2}$ \\ ${ }^{1}$ Assistant Professor, ${ }^{2}$ Associate Professor, Dept. of Orthopaedics, Rajarajeswari Medical College and Hospital, Bangalore, \\ Karnataka, India
}

*Corresponding Author:

Email: dravicrp22@gmail.com

\begin{abstract}
Increase of in number of joint replacement has challenged orthopaedic surgeons with task of balancing the complications of venous thrombo embolism on one side and adverse effects of prophylactic agents like bleeding on other side. Despite numerous studies and extensive clinical experience, the ideal method of venous thromboembolism prophylaxis remains controversial. Aspirin as an agent for deep vein thrombosis prophy laxis after total joint Arthroplasty is safe, inexpensive, does not require blood parameters monitoring and easy to use with excellent patient compliance supplemented with mechanical prophylaxis in the form of DVT pumps, early mobilization and CPM further reduces the chances of DVT. In this view we have decided to study the incidence of DVT in joint replacement patients treated with asiprin and mechanical devices alone, which as shown satisfactory results with non of our patients developing pulmonary embolism with incidence of DVT in our study is as low as $1 \%$.
\end{abstract}

Keywords: Deep vein thrombosis, Hip replacement, Knee replacement, Aspirin, Mechanical devices.

\section{Introduction}

Increase in number of joint replacement has challenged orthopaedic surgeons with task of balancing the complications of venous thromboembolism on one side and adverse effects of prophylactic agents like bleeding on other side. Despite numerous studies and extensive clinical experience, the ideal method of venous thromboembolism (VTE) prophylaxis remains controversial.

However the threat of thromboembolic events has reduced during the past decade, ${ }^{16,26,27}$ the risk of fatal pulmonary embolism is around $0.1 \% .^{3-6}$ This decreased risk is mainly because of the change in post operative fast tracking protocol like rapid mobilization, less operative time, regional anaesthesia, mechanical compression devices. In spite of low risk there is always a debate regarding the deep vein thrombosis (DVT) prophylaxis and many agents that lower the incidence of DVT are associated with increased risk of bleeding complications.

Guidelines for DVT Prophylaxis proposed in ACCP, ${ }^{23}$ a study involving multiple specialities mainly pulmonologist is mainly on presence or absence of DVT and have ignored the adverse events of the agents. Hence the reduction in DVT as a final clinical measure will not balance the risk analysis of the safety and efficacy of the agent used.

Aspirin as an agent for DVT prophylaxis after total joint arthroplasty is safe, inexpensive, does not require blood parameters monitoring and easy to use with excellent patient compliance. The usage of aspirin is mainly on the belief that prophylaxis is a must to reduce the risk of pulmonary embolism and not DVT. Overall the inference is that DVT is not a marker for pulmonary embolism, since all patients with a DVT do not end up in Pulmonary embolism (PE) and not all with PE have detectable DVT. . $^{13,36-38}$

But aspirin is not thought to be as effective as other chemophrophylactic agents and no clinical trials to compare aspirin with others drugs to prove its efficacy. More recent publications with larger numbers have suggested that the risk of VTE following either aspirin or inject able prophylaxis against VTE (LMWH/fondaparinux) is equivalent ${ }^{10}$

Hence we studied the efficacy of aspirin combined with mechanical devices as prophylaxis in total joint arthroplasty which was evaluated by colour Doppler study during pre op, post op and at 6 weeks follow up.

\section{Materials and Methods}

This is prospective study where we included 247 patients undergoing total joint arthroplasty (hip and knee), both primary and revision, unilateral and bilateral between August 2014 to September 2017 in our institute after obtaining approval from institutional review board and written consent. However patients with pre-existing DVT or history of previous thromboembolism, those on anti-coagulant for ischemic heart disease, cerebrovascular accident etc., documented bleeding within 3 months prior to surgery like cerebral hemorrhage, GI bleed etc was excluded.

A protocol was formulated, which includes patients details like demographic parameters, co-morbidities, any prior surgeries, any medications, nature and duration of surgery, type of anaesthesia, any complications (intra and post-op) Aspirin related complications such as gastric intolerance, ecchymosis, bleeding from wound, etc. were noted.

All patients admitted one day prior to surgery and pre op Doppler done same day. All operated under regional anaesthesia with single dose of tranaxamic 
acid injection just before surgery. All diabetic patients received

Insulin for blood sugar control during hospital stay later converted back to their pre op status. All hypertensive patients received their regular medicine as per schedule. Mechanical prophylaxis with IPCD (Intermittent Pneumatic Compression Devices) and CPM (continuous passive motion) started on immediate post-operative period. Mobilization started from $1^{\text {st }}$ post op day after disconnecting the IPCD, which is tapered off in next 24-48 hours. Tab Aspirin 150mg PO once daily started 12-24 hours post-surgery and continued for up to 4 weeks post-discharge. If any signs of persistent ooze from surgical wound or progressive increasing ecchymosis noted, aspirin was stopped for few days and observed. In cases clinically suspicious of DVT, Doppler Scan was done immediately otherwise usually done before discharge on $5^{\text {th }}$ or $7^{\text {th }}$ post operative day. Clinical features suggestive of pulmonary embolism were also looked for, such as chest pain, tachycardia, hypoxemia, ECG changes, further evaluated with Doppler scan, serum d-dimer levels, $\mathrm{ABG}$ and if needed CT angiography at any point of post-operative period. Established cases of deep vein thrombosis were started on $1 \mathrm{mwh} 1.5 \mathrm{mg} / \mathrm{kg}$ body weight once daily along with Warfarin sodium therapy (usually within 72 hours of commencing lmwh). LMWH continued for 3-5 days and until a therapeutic oral anticoagulant effect has been achieved (International Normalization Ratio 2.0 to 3.0).In staged bilateral cases done at one week interval, Tab Aspirin $150 \mathrm{mg}$ was stopped two days before the second surgery and restarted on day 1 again and continued for 4 weeks post discharge. Patients were seen in the outpatient clinic one month post surgery and again Doppler study done to rule out DVT.

\section{Results}

Out of 247 patients 190 were TKR and 57 were THR cases. Among TKR, 115 are females and 75 are males. 69 cases are Bilateral TKR. 4 were revision TKR and rest primary. Among THR 32were males and 25 were females. Related to co-morbidities, 78 patients had hypertension, 116 had diabetes mellitus and 10 patients had hypothyroidism. Mean stay in hospital was 6 days ranging from 4 days to 10 days. Surgical duration range from $45 \mathrm{~min}$ to $1 \mathrm{hr} 15 \mathrm{~min}$ with tourniquet. Mean intra op blood loss $20 \mathrm{ml}$ ranging from $10 \mathrm{ml}$ to $30 \mathrm{ml}$.

Out of 3 DVT encountered in our study, 2 cases were proximal with one symptomatic and another asymptomatic one in THR \& one in TKR. Only one asymptomatic distal DVT in a bilateral staged TKR noted.

In this study with aspirin as prophylactic agent we noticed, eleven patients had echymosis, one had persistent wound ooze. All settled uneventfully with compression bandage and withholding aspirin for few days. None of the patients required re-exploration for hematoma evacuation or ended up in infection.

Table 1: Venous thrombo embolism events

\begin{tabular}{|l|c|}
\hline $\begin{array}{c}\text { Asymptomatic DVT } \\
\text { (pre operatively) }\end{array}$ & $\begin{array}{c}\mathbf{4} \text { (excluded from } \\
\text { study) }\end{array}$ \\
\hline $\begin{array}{l}\text { Asymptomatic DVT(post } \\
\text { operatively) }\end{array}$ & 2 \\
\hline Symptomatic DVT & 1 \\
\hline Symptomatic PE & 0 \\
\hline Fatal PE & 0 \\
\hline Proximal DVT & 2 \\
\hline Distal DVT & 1 \\
\hline In THR(57) & 1 \\
\hline In TKR(190) & 2 \\
\hline
\end{tabular}

Table 2: Body mass index

\begin{tabular}{|l|c|}
\hline Range of BMI & Number of patients \\
\hline$<25$ & 113 \\
\hline $25-30$ & 67 \\
\hline $30-35$ & 41 \\
\hline $35-40$ & 17 \\
\hline$>40$ & 09 \\
\hline
\end{tabular}

Table 3: Advers events of Aspirin

\begin{tabular}{|l|c|}
\hline Ecchymosis & 11 \\
\hline Persistent soakage & 01 \\
\hline Hematoma collection & 00 \\
\hline $\begin{array}{l}\text { Re-exploration for } \\
\text { hematoma }\end{array}$ & 00 \\
\hline Infection & 00 \\
\hline
\end{tabular}

\section{Discussion}

In our study incidence of DVT is $0.3 \%$ that is 3 out of 247 patients. Such low incidences are reported in study by Kim and $\operatorname{Suhn}^{13}$ (1988), where they found only $10 \%$ of 146 Korean patients who underwent uncemented THA had DVT and Atichartakarn ET al ${ }^{14}$ (1988) did not find any cases of DVT in a study of 19 Thai THA patients. In a study by V. Jain ET $\mathrm{al}^{16}(2004)$ incidence of DVT was $0 \%$ out of 50 hip cases. But in study by Agarwal et al, ${ }^{19}$ high incidence of DVT shown is based on invasive venography which picks up very tiny insignificant thrombus of no clinical significance.

We noticed two cases of proximal and one case of distal DVT. Both proximal were in THR and one distal in TKR, supporting literature that proximal DVT are more common in THR and distal DVT in TKR. Two cases of DVT were Asymptomatic, detected with routine Doppler study and the one symptomatic proximal DVT, diagnosis picked up clinically later confirmed with Doppler scan. No incidence of asymptomatic, symptomatic and fatal PE noted in this study. Similar results are observed in studies by Lawrence D Dorr et $\mathrm{al}^{20}$ (2007), with overall incidence of DVT was $5.6 \%$ and symptomatic DVT of $0.4 \%$ and Non fatal PE of $0.25 \%$ in a series of 1176 patients and 
by Lachiewicz and Soileau ${ }^{21}$ (2006) in their study on 1032, overall incidence of DVT was $3.9 \%$ and symptomatic DVT $0.4 \%$, like wise by Lotke et $\mathrm{al}^{22}$ (2006) incidence of symptomatic DVT in 3473 TKR cases was $0.2 \%$ and Non fatal PE of $0.26 \%$.

Since not all patients with DVT end up with PE, just presence of DVT is not an indication for inject able anticoagulants. Studies comparing incidence of DVT between two prophylactic agents had under reported the side effects of agent like persistent oozing, hematoma collection, prolonged hospitalstay, infection and others, which all significantly affect the outcome of joint replacement surgery. However our incidence of DVT was also low by Doppler study, which was done to prove the point that even if LMWH is not given incidence DVT is negligible.

LMWH used by Arthroplasty surgeons is due to fear factor of VTE and to avoid litigation issues as it is considered has standard of care based on ACCP \& SCIP guidelines..$^{23}$ In our study we have taken symptomatic distal and asymptomatic proximal DVT as end point, unlike ACCP guidelines ${ }^{23}$ which focused on the prevention of the overall rate of VTE. Its guidelines were based on a review of prospective, randomized studies involving mainly non orthopaedic group. According to which major bleeding episode to be considered has complication are, death or life threatening clinical event, bleeding confirmed to be retroperitoneal, intracranial or intraocular, transfusion of more than two units of packed blood cells or whole blood, or a decrease in haemoglobin greater than $20 \mathrm{~g} / \mathrm{l}$ compared with the relevant pre-operative level. ${ }^{24-25}$ Persistent wound oozing, hematoma collection, progressing ecchymosis, re-exploration to evacuate the collection which are enough events to get a replaced joint infected is not given importance. Further dose \& timing adjustment of LMWH is required to prevent epidural hematoma in neuraxial anaesthesia. Particularly in revision cases and in complex primary case with excessive tissue desiccation use of LMWH may be disastrous with risk of developing compartment syndrome.

Aspirin is an effective antiplatelet agent, acts by permanently inactivating the cyclooxygenase activity of prostaglandin $\mathrm{H}(\mathrm{COX}-1)$ with dose as low as 30 to 50 mg per day. Since there are very few studies on a large cohort using aspirin as a chemo prophylactic agent for DVT, it is considered less effective and lacks literature support to defend legally. Compared to LMWH it is less expensive, oral preparation easily taken by patient themselves without need of nursing staff and can be taken at home without hassles of blood parameter monitoring which greatly enhanced the compliance. Most important is less bleeding and wound related complications. Has in our study, incidences of minor bleeding complications are $4.7 \%$ \& major bleeding complications were $0 \%$. Similar low incidence of bleeding complications and VTE are noted in studies by
Lotke and colleagues ${ }^{29}$ reported on 2800 consecutive primary TKAs in patients treated with aspirin and mechanical prophylaxis, demonstrating a low rate of bleeding complication and a fatal PE risk of $0.1 \%$., aspirin is considered as safe and effective agent in Pulmonary Embolism Prevention (PEP) trial, ${ }^{30}$ Bozic et $\mathrm{al}^{31}$ retrospectively compared rates of VTE, bleeding, infection and mortality in 93840 patients undergoing knee replacement using one of three pharmacological agents (aspirin, $\mathrm{n}=4719$; $\mathrm{LMWH} /$ fondaparinux, $\mathrm{n}=37$ 198; warfarin, $\mathrm{n}=51$ 923). Once adjusted for case mix there were no significant differences for any outcomes between the aspirin and LMWH /fondaparinux groups. In a Freedman et $\mathrm{al}^{32}$ study in 2000 , showed lowest rate of minor \& major bleeding complications with aspirin compared to LMWH, warfarin \& placebo.

Intermittent pneumatic compression device is an effective adjuvant to chemoprophylaxis, which prevents the development of clots in distal limb by maintaining venous drainage passively. A multicenter RCT published by Colwell et $\mathrm{al}^{33}$ in 2010 showed a mobile IPC achieved equivalent incidence of proximal and distal DVT and PE with no deaths and a much lower bleeding rate (1.3\% versus $4.3 \%)$.

In our study we used Doppler ultrasound has the diagnostic tool for detection of DVT. This is noninvasive and provides good sensitivity (89\%) and specificity (100\%) for detecting proximal DVT as well. ${ }^{12}$ Unlike Venography which is invasive, technically demanding and associated with contrast related complications, venous Doppler study is non invasive, quick and safe. Its efficacy in detecting both proximal and distal DVT is proven by various studies like, in a Japanese study, ${ }^{34} 9 \%$ of 33 patients undergoing THA detected having proximal DVT based on Doppler ultrasonography of both lower limbs, In an Indian study, ${ }^{35} 2(1.3 \%)$ of 60 patients developed proximal DVT after THA, assessed by Doppler ultrasonography of both lower limbs.

\section{Conclusion}

Incidence of DVT is very low among population undergoing elective surgical procedures like joint replacement surgery. Low dose Aspirin with intermittent pneumatic compression device is a less aggressive, less expensive \& more cost effective prophylactic method without added risk of excessive bleeding. LMWH is best agent in treatment of symptomatic DVT and is been overused has a prophylactic agent leading to unnecessary complications and added cost. Doppler ultrasonography is an ideal non invasive diagnostic tool for detection of both proximal and distal DVT. We need to aim at prevention of symptomatic DVT and its subsequent complications of PE, and not focus merely to reduce the incidence of asymptomatic, clinically insignificant DVT. Importance should be given to patient selection, multimodal approach, meticulous surgical techniques, 
aggressive physiotherapy and proper patient education rather than implementing guidelines to avoid legal is sues.

\section{References}

1. Memtsoudis SG, Pumberger M, Ma Y, Chiu YL, Fritsch G, Gerner P, et al. Epidemiology and risk factors for perioperative mortality after total hip and knee arthroplasty. J Orthop Res 2012;30:1811-1821.

2. Comp PC, Spiro TE, Friedman RJ, et al. Prolonged enoxaparin therapy to prevent venous thromboembolism after primary hip or knee replacement. Enoxaparin Clinical Trial Group. J Bone Joint Surg Am 2001;83(3):336-45.

3. Douketis JD, Eikelboom JW, Quinlan DJ, et al. Short duration prophylaxis against venous thromboembolism after total hip or knee replacement: a meta-analysis of prospective studies investigating symptomatic outcomes. Arch Intern Med 2002;162(13):1465-71.

4. Brookenthal KR, Freedman KB, Lotke PA, et al. A metaanalysis of thromboembolic prophylaxis in total knee arthroplasty. J Arthroplasty 2001;13(3):293-300.

5. Freedman KB, Brookenthal KR, Fitzgerald RH Jr, et al. A meta-analysis of thromboembolic prophylaxis following elective total hip arthroplasty. JBone Joint Surg Am 2000;82(7):929-38.

6. Larson CM, MacMillan DP, Lachiewicz PF. Thromboembolism After total knee arthroplasty: intermittent pneumatic compression and aspirin prophylaxis. J South Orthop Assoc 2001;10(3):155-63 [discussion: 163].

7. Lieberman JR, Hsu WK. Prevention of venous thromboembolic disease after total hip and knee arthroplasty. J Bone Joint Surg Am 2005;87(9):2097-112.

8. Haas SB, Insall JN, Scuderi GR, Windsor RE, Ghelman B. Pneumatic sequential-Compression boots compared with aspirin prophylaxis of deep-vein thrombosis After total knee arthroplasty. J Bone Joint Surg [Am ] 1990;72A:27-31.

9. McKenna R, Galante J, Bachmann F, et al. Prevention of venous thromboembolism After total knee replacement by high-dose aspirin or intermittent calf and thigh Compression. Br Med J 1980;280:514-517.

10. Bozic KJ, Vail TP, Pekow PS, et al. Does aspirin have a role in venous thromboembolism? Prophylaxis in total knee arthroplasty patients? J Arthroplasty 2010;25:10531060.

11. Poultsides LA, Gonzalez Della Valle A, Memtsoudis SG, et al. Meta-analysis of Cause of death following total joint replacement using different thromboprophylaxis Regimens. J Bone Joint Surg [Br] 2012;94-B:113-121.

12. Cronan JJ, Dorfman GS, Scola FH, Schepps B, Alexander J. Deep venous thrombosis: US assessment using vein compression. Radiology 1987;162:191-4.

13. Kim YH, Suh JS. Low incidence of deep-vein thrombosis after cement less total hip replacement. J Bone Joint Surg Am 1988;70:878-82.

14. Atichartakarn V, Pathepchotiwong K, Keorochana S, Eurvilaichit C. Deep vein thrombosis after hip surgery among Thai. Arch Intern Med 1988;148:1349-53.

15. Ko PS, Chan WF, Siu TH, Cheng A, Lee OB, and Lam JJ. Duplex ultrasonography after total hip or knee arthroplasty. Int Orthop 2003;27:168-71.

16. Jain V, Dhal AK, Dhaon BK, Pradhan G. Deep vein thrombosis after total hip arthroplasty in Indian patients with and without enoxaparin. Journal of Orthopaedic Surgery 2004;12(2):173-177.
17. Angral R1, Islam MS2, Kundan S3: Incidence of deep vein thrombosis and justification of chemoprophylaxis in Indian patients: A prospective study; Bangladesh Med Res Counc Bull 2012;38:67-71.

18. Smit Shah et al/ International Journal of Biomedical and Advance Research 2015;6(01): 15-19; Prevalence of deep vein thrombosis with lower limb trauma patients.

19. Agarwala S, Bhagwat A, Modhe JM, Dastur FD, Patil S. Incidence of deep vein thrombosis in Indian patients A prospective study in 104 patients by: Indian journal of Orthopaedics. January 2005 Volume 39: Number 1:P. 5558.

20. Dorr L, Gendelman V, Maheshwari A, Boutary M, Wan Z, Long W. Multimodal thromboprophylaxis for total hip and knee arthroplasty based on risk assessment. J Bone Joint Surg Am 2007;89:2648-57.

21. Lachiewicz PF, Soileau ES. Multimodal prophylaxis for THA with mechanical compression. CORR 2006;453:225-30.

22. Lotke PA, Lonner JH. The benefit of aspirin chemoprophylaxis for thromboembolism after total knee arthroplasty. CORR.2006;452:175-80.

23. Haas S. Prevention of venous thromboembolism: recommendations based on the International Consensus and the American College of Chest Physicians Sixth Consensus Conference on Antithrombotic Therapy. Clin Appl Thromb Hemost 2001;7:171-177.

24. Geerts WH, Pineo GF, Heit JA, et al. Prevention of venous thromboembolism: the Seventh ACCP Conference on Antithrombotic and Thrombolytic Therapy. Chest 2004;126(Suppl):338S-400S.

25. Geerts WH, Bergqvist D, Pineo GF, et al. Prevention of venous thromboembolism: American College of Chest Physicians Evidence-Based Clinical Practice Guidelines (8th Edition). Chest 2008;133(Suppl):381S-453S.

26. Keeney JA, Clohisy JC, Curry MC, Maloney WJ. Efficacy of combined modality Prophylaxis including short-duration warfarin to prevent venous thromboembolism After total hip arthroplasty. $J$ Arthroplasty 2006;21:469-475.

27. Novicoff WM, Brown TE, Cui Q, et al. Mandated venous thromboembolism prophylaxis: Possible adverse outcomes. J Arthroplasty 2008;23:15-19.

28. Jacobs JJ, Mont MA, Bozic KJ, et al. American Academy of Orthopaedic Surgeons Clinical practice guideline on: preventing venous thromboembolic disease in Patients undergoing elective hip and knee arthroplasty. J Bone Joint Surg [Am] 2012;94-A: 746-747.

29. Lotke, P. A.: Thromboembolic disease: a critical review. Bull. Am. Acad. Orthop. Surg.1989:37:20-21.

30. PEP Trial Collaborative Group. Prevention of pulmonary embolism and deep vein thrombosis with low dose aspirin: Pulmonary Embolism Prevention (PEP) trial. Lancet 2000; 355(9212):1295-302.

31. Bozic KJ, Vail TP, Pekow PS, et al. Does aspirin have a role in venous thromboembolism prophylaxis in total knee arthroplasty patients? J Arthroplasty 2010;25:105360.

32. Freedman KB, Brookenthal KR, Fitzgerald RH, Williams S, Lonner JH. A Meta-Analysis of Thromboembolic Prophylaxis Following Elective Total Hip Arthroplasty. The Journal of Bone and Joint Surgery, Vol 82-A, No 7 July 2000.

33. Colwell CW, Jr, Froimson MI, Mont MA, et al. Thrombosis prevention after total Hip arthroplasty: a prospective, randomized trial comparing a mobile compression Device with low-molecular-weight heparin. J Bone Joint Surg [Am] 2010; 92-A:527-535. 
34. Sudo A, Sano T, Horikawa K, Yamakawa T, Shi D,

Uchida A. The incidence of deep vein thrombosis after hip and knee Arthroplasties in Japanese patients: a prospective study. J Orthop Surg (Hong Kong) $2003 ; 11: 174-7$.

35. Jain V, Dhaon BK, Jaiswal A, Nigam V, Singla J. Deep vein thrombosis after total hip and knee arthroplasty in Indian patients. Postgrad Med J 2004;80:729-31.

36. Lotke PA, Ecker ML, Alavi A, Berkowitz H. Indications for the treatment of deep venous thrombosis following total knee replacement. J Bone Joint Surg [Am] 1984;66A:202-8.

37. Lotke PA, Steinberg ME, Ecker ML. Significance of deep venous thrombosis in the lower extremity after total joint arthroplasty. Clin Orthop 1994;299:25-30.

38. Lotke PA, Wong RY, Ecker ML. Asymptomatic pulmonary embolism after total knee replacement. Orthop Trans 1986;10:490. 